\title{
DIABETES Y EMBARAZO (II PARTE): MANEJO
}

Dr. ARTURo EsQuivel Grillo

Profesor Asociado ObStetricia U.C.R.

Jefe del Servicio de Obstetricia del H.C.G.

\section{PALABRAS CLAVE: DIABETES, EMBARAZO, ACTUALIZACION TERAPEUTICA.}

El manejo de la diabetes durante el embarazo puede enfocarse desde varios puntos de vista: a)materno y fetal; b)atendiendo al momento cronológico del embarazo, o sea, anteparto, transparto y postparto y c) en relación con criterios de mayor trascendencia, como es el relativo a la severidad del cuadro clínico (1-2). Este es el que se aplica en la tabla No.8 de la primera parte de este artículo y que utilizaremos en la presente revisión para describir el manejo individualizado.

\section{MANEJO DE PACIENTES CON INTOLERANCIA GESTACIONAL A LOS CARBOHIDRATOS.}

Estas se manejan a base de dieta (3). Esta debe ser calculada a base de las calorías requeridas según el peso ideal de la paciente, expresado en kilogramos. El número de calorías requerido por cada kilo de peso corporal es 35 . Por consiguiente, este factor es multiplicado por el peso ideal pregestacional, en kilos, para obtener el total de calorías que requiere la paciente en 24 horas (Tabla Nol.).

TABLA NO. 1 APORTE CALORICO Y EMBARAZO

\begin{tabular}{lcc}
\hline Talla (cms) & $\begin{array}{l}\text { Peso Ideal } \\
\text { Pre-Embarazo }\end{array}$ & $\begin{array}{c}\text { Calorías Recomendadas } \\
(35 \mathrm{Kcal} / \text { Kilo })\end{array}$ \\
\hline 147 & 48,5 & 1701 \\
150 & 50 & 1750 \\
152 & 51 & 1799 \\
155 & 52,7 & 1845 \\
158 & 54 & 1894 \\
160 & 55,9 & 1957 \\
163 & 58,2 & 2037 \\
165 & 60 & 2100 \\
168 & 61,8 & 2163 \\
170 & 63,6 & 2226 \\
173 & 65,7 & 2500 \\
175 & 67 & 2356 \\
178 & 69 & 2419
\end{tabular}

Se aconseja agregar 300 calorías por el embarazo mismo, especialmente en la segunda mitad, cuando realmente aumentan los requerimientos calóricos. Del total de las calorías un $50 \%$ deben de ser carbohidratos. Las calorías se deben repartir en: 3 comidas principales y de 1 a 3 meriendas para mantener niveles de glicemia estables. Con este aporte calórico la paciente con intolerancia a los carbohidratos debería ganar, como máximo, 15 libras durante el embarazo. Si es obesa, inclusive podría no ganar peso y hasta perder, sin consecuencias adversas (4-5).

La presencia de cetonuria sugiere que la paciente está usando su propia grasa más que los carbohidratos de la propia dieta para suplir sus necesidades calóricas. En una paciente obesa, una leve cetonuria no es un signo ominoso. Si la cetonuria es marcada (más de +++ ) se debe incrementar el consumo de carbohidratos. Si este llega a producir hiperglicemia post-prandial se requerirá terapia insulínica. El control dietético es más efectivo en la prevención de la macrosomia fetal si la madre es mantenida justamente sobre el nivel del umbral cetonúrico (6).

La glicemia se debe controlar en ayunas y dos horas postprandial; la primera no debe exceder de $105 \mathrm{mg}$./dl. Las post-prandiales no deben ser mayores de $120 \mathrm{mg}$./ dl. Si la dieta no logra este objetivo metabólico, se debe recurrir a la insulina (3).

Como se describió en la clasificación de la diabetes, en el primer artículo, este grupo de pacientes con intolerancia gestacional a los carbohidratos, se subdividen en dos subgrupos catalogados de alto y bajo riesgo, que aquí analizaremos independientemente.

\section{IA. PACIENTES CON INTOLERANCIA GESTACIONAL A LOS CARBOHIDRATOS, DE BAJO RIESGO.}

Generalmente, no hacen complicaciones maternas ni fetales, por lo que no requieren vigilancia especial ni monitoreo fetal anteparto. Con solo el auto control de movimientos fetales por la paciente, previamente instruida al respecto, es suficiente. Si se dispone de estimulación vibro-acústica sí es conveniente aplicar ésta en cada visita prenatal. Por lo que respecta al parto, estas pacientes de bajo riesgo deben ser manejadas de manera similar a la embarazada normal no diabética, permitiendo que su gestación llegue a término y esperando el inicio de labor de parto espontánea. 


\section{IB PACIENTES CON INTOLERANCIA GESTACIONAL A LOS CARBOHIDRATOS, VE ALTO RIESGO.}

Estas sí requieren una atención especial, particularmente en cuanto a la vigilancia fetal

IB-1 Vigilancia Fetal: Esta debe iniciarse a partir de la semana 34 de gestación por alguno de los métodos indicados en la Tabla No.2: Prueba sin Esfuerzo (NST), Prueba de Esfuerzo (CST) y Perfil Biofísico Fetal (PBF).

TABLA NO. 2

VIGILANCIA FETAL

\begin{tabular}{lll}
\hline Método & Frecuencia & Valoración Básica \\
\hline NST & $2 \mathrm{v} \mathrm{p} /$ semana semanal & $\begin{array}{l}\text { Reactividad fetal } \\
\text { Desaceleraciones } \\
\text { CST }\end{array}$ \\
semanal & Hipomovilidad fetal \\
PBF & semanal &
\end{tabular}

El NST es la más utilizada de estas pruebas. No obstante, la CST probablemente, proporciona la evidencia más temprana de sufrimiento fetal, porque las desaceleraciones tardías aparecen antes que los cambios en reactividad o que la hipomovilidad fetales.

IB- 2 Vigilancia Materna: Desde el punto de vista materno, las pacientes deben manejarse fundamentalmente, con control calórico y dietético, al igual que el grupo de las intolerantes a la glucosa de bajo riesgo. Para tal propósito se debe efectuar la valoración de los niveles de glicemia en ayunas y postprandiales para establecer el perfil glicémico según se indica en la Tabla No.3.

TABLA No.3

VALORACION DE NIVELES DE GLICEMIA

(PERFIL GLICEMICO)

-En ayunas.

-2 horas postdesayuno.

-2 horas postalmuerzo

-2 horas postcena

Las pacientes, que a pesar de la dieta presentan glicemias persistentemente elevadas, requieren insulina, cuya dosis y tipo farmacológico dependen de la severidad y momento del desajuste metabólico (Tablas No. 2,4) y de las características de la insulina utilizada (Tablas No.3,4,5 y 6).

TABLANO.4

INSULINA

\begin{tabular}{ll}
\hline Tipo & Dosis inicial \\
Regular & $5-10 \mathrm{U}$ \\
Lenta(NPH) & $5-15 \mathrm{U}$ \\
\hline
\end{tabular}

TABLANO.5

\begin{tabular}{lccc}
\hline Tipos & $\begin{array}{c}\text { Inicio de Acción } \\
\text { (horas) }\end{array}$ & $\begin{array}{c}\text { Efecto Máximo } \\
\text { (horas) }\end{array}$ & $\begin{array}{c}\text { Duración } \\
\text { (horas) }\end{array}$ \\
Regular & $1 / 4$ & $4-6$ & $6-8$ \\
NPH & 3 & $8-12$ & $18-24$ \\
\hline
\end{tabular}

El control de la hiperglicemia, particularmente post-prandial, es fundamental en la prevención de la macrosomia fetal (7). Estas pacientes no tienen tendencia a cetoacidosis ni a hipoglicemia. No deben usarse hipoglicemiantes orales durante el embarazo porque provocan hiper-insulinemia fetal prolongada e hipoglicemia neonatal. También pueden agravar la hiperbilirrubinemia neonatal por entrar en competencia con los sitios fijadores de albúmina.

TABLA No.6

ADMINISTRACION DE INSULINA

\begin{tabular}{|c|c|c|c|}
\hline Hiperglicemia & Tipo & Dosis Inicial & Momento de aplicación \\
\hline En ayunas & NPH & $10-15 \mathrm{U}$ & al acostarse \\
\hline Post-prandial única & Regular & $10 \mathrm{U}$ & $\begin{array}{l}\text { Antes de la } \\
\text { respectiva comida }\end{array}$ \\
\hline $\begin{array}{l}\text { Post-prandial } \\
\text { múltiple }\end{array}$ & $\begin{array}{l}\text { NPH } \\
\text { Regular }\end{array}$ & $\begin{array}{l}1015 \mathrm{U} \\
5 \mathrm{U}\end{array}$ & En ayunas \\
\hline
\end{tabular}

Por lo que se refiere al parto, éste debe ocurrir entre las semanas 38 y 40 de gestación, confirmada clínica y sonográficamente. El problema que se presenta es que muchas pacientes, en estas circunstancias, tienen un cuello uterino inmaduro por lo que la inducción con oxitocina es menos exitosa y más predisponente a la operación cesárea. En este sentido, es preferible esperar la labor espontánea, pues tiene mejor pronóstico en cuanto a la vía vaginal, a pesar de que el feto experimente un aumento de peso adicional. Por otra parte, la maduración cervical con derivados prostaglandínicos puede obviar la inmadurez y facilitar el parto vaginal, siendo esta la conducta preferida en la actualidad. En las pacientes que están recibiendo insulina, ésta debe ser suspendida durante la labor, parto o cesárea. La glicemia debe controlarse cada cuatro 'horas y corregirse con insulina regular cualquier desviación de la normalidad.

En las pacientes intolerantes a la glucosa, con frecuentes hiperglicemias o que fueron diagnosticadas como tales antes de la semana 24 de la gestación, debe repetirse la curva de tolerancia a la glucosa postparto, ya que de $9 \%$ a $44 \%$ de ellas tienen diabetes mellitus (8). La gran mayoría se normalizan postparto. Sin embargo, deben ser informadas de que tienen un $50 \%$ de posibilidades de Llegar a ser diabéticas tipo II en la quinta década de su vida, pronóstico que podrían prevenir o retardar si controlan la dieta, el apetito y hacen ejercicio $(9,10)$. 


\section{MANEJO DE PACIENTES EMBARAZADAS CON DIABE-} TES TIPO II O NO INSULINO DEPENDIENTES.

Estas pacientes ya tienen su diagnóstico antes del embarazo, así como también su control dietético y calórico. De manera muy similar al grupo anterior con intolerancia a los carbohidratos, estas pacientes, desde el punto de vista materno, se siguen manejando inicialmente con solo control dietético. Si a pesar de ello, ocurren frecuentes hipoglicemias, debe instaurarse la insulinoterapia. Desde el punto de vista fetal, debe iniciarse la vigilancia biofísica desde la semana 34 de gestación de preferencia con el CST. Si la diabetes se mantiene compensada, como es lo usual, el embarazo se dejará evolucionar hasta la semana 38-40.

\section{MANEJO DE PACIENTES EMBARAZADAS CON DIABETES TIPO $1 \mathrm{~V}$ INSULINO DEPENDIENTE, SIN DAÑO ORGANICO.}

También estas pacientes tiene su diagnóstico y su insulino-terapia y dieto-terapia establecidas antes del embarazo. Los objetivos del manejo se resumen en la tabla No. 7.

TABLA No. 7

OBJETIVOS DEL MANEJO DE LA PACIENTE

EMBAPAZADA CON DIABETES TIPO I

\begin{tabular}{ll} 
I) Período Gestacional & Objetivos \\
\hline Madre & Control Metabólico \\
Feto & $\begin{array}{l}\text { Detección de Embriopatía Diabética } \\
\text { Detección de Macrosomia Detección } \\
\text { Detección de Hipoxia }\end{array}$
\end{tabular}

2) Período del Parto

\begin{tabular}{ll}
\hline Madre & $\begin{array}{l}\text { Control Metabólico } \\
\text { Momento del Parto } \\
\text { Vía del Parto }\end{array}$ \\
Feto & Monitoreo Continuo \\
\hline 3) Periodo Post-Parto & Control Metabólico
\end{tabular}

Control Metabólico en Embarazo. Se debe medir diariamente la glicemia en ayunas y 2 horas postprandial, mediante 1 utilización de las tiras o máquinas destinadas a ese propós ). Si hay alteraciones casi siempre se deben a indisciplina en la dieta. Debe preguntarse sobre la calidad y cantidad de los alimentos consumidos e insistir en la trascendental importancia de cumplir la ingesta calórica asignada y la terapia insulínica a la dosis y hora exactas (11).
TABLA No.8

VALORACION DE EFECTOS DE INSULINA SOBRE NIVELES DE GLICEMIA

\begin{tabular}{lll}
\hline Tipo de Insulina & Aplicación & Repercusión en Glicemia \\
& & \\
Regular & antes desayuno & 2 horas postdesayuno \\
Regular & antes cena & 2 horas postcena \\
NPH & matutina & 2 horas postalmuerzo \\
NPH & nocturna & en ayunas \\
\hline
\end{tabular}

La decisión de efectuar cambios en la dosis de insulina, debe basarse no solo en los niveles de glicemia, sino que hay que asegurarse del tipo, las dosis y la hora a que la paciente recibe la insulina (Tabla No. 8), así como del número, tamaño, tipo y hora de las comidas que recibe. Solo después del análisis conjunto de todos estos factores, se justificará el cambio de dosis $(12,13)$.

1-A. DETECCION DE EMBRIOPATIA DIABETICA. En la paciente portadora de diabetes, previa al embarazo, es obligatorio valorar la hemoglobina glicosilada o hemoglobina Alc poco después de ocurrida la concepción. Esta fracción hemoglobinica es glicosilada, dependiendo de la concentración de azúcar, de una manera directa, sin intervención de enzimas. Si la prueba es efectuada de 4 a 6 semanas después de la concepción, reflejará los niveles de glicemia de la paciente en el período pre y postconcepcional (14-15-16). Si los niveles son elevados, implican un importante riesgo de malformaciones fetales como se indica en las Tablas 9 , 10 y 11 .

TABLA 9 INCIDENCIA DE ANOMALIAS CONGENITAS MAYORES

\begin{tabular}{ll}
\hline $\begin{array}{l}\text { Embarazadas } \\
\text { Normales }\end{array}$ & $\begin{array}{l}\text { Embarazadas } \\
\text { Diabéticas }\end{array}$ \\
$1 \%$ & $7,5 \%-13 \%$ \\
\hline
\end{tabular}

TABLANo.10

\section{PRINCIPALES ANOMALIAS CONGENITAS MAYORES EN INFANTES DE MADRES DIABETICAS}

\section{-Neurales}

Anencefalia, encéfalocele, espina bífida, holoprosencefalia

\section{-Cardíacas}

Transposición de grandes vasos, defectos del tabique ventracular coartación aórtica

\section{-Esqueléticas}

Regresión caudal senales Agenesia renal duplicación ureteral

-Gastrointestinales

Atresia anal y rectal 
TABLA No.11

FRECUENCIA DE ANOMALIAS FETALES CON

HEMOGLOBINA GLICOSILADA ALTERADA

Hemoglobina Glicosilada $=20 \%$ a $25 \%$ de probabilidad de mayor de $8,5 \%$ anomalías

Si la hemoglobina glicosilada está alterada, la paciente deberá seguir la secuela de valoraciones de embriopatías diabéticas que se encuentra en la tabla No. 12.

TABLA. 12

DETECCION DE EMBRIOPATIAS DIABETICAS

\begin{tabular}{|c|c|c|}
\hline Exámenes & Momento & Detección \\
\hline ultrasonido & $8-10$ sem. & $\begin{array}{l}\text { anencefalia } \\
\text { holoprosencefalia } \\
\text { emb. anembriónico }\end{array}$ \\
\hline $\begin{array}{l}\text { Alfa-fetoproteína } \\
\text { sérica materna }\end{array}$ & $16 \mathrm{sem}$ & defectos del tubo neural \\
\hline Ultrasonido & $18-20$ sem. & $\begin{array}{l}\text { malformaciones fetales } \\
\text { antes no detectables }\end{array}$ \\
\hline EKG fetal & $24-26$ sem. & cardiopatías \\
\hline
\end{tabular}

Las anomalías más frecuentes afectan el corazón y el sistema nervioso central (17). Las más comunes son: anencefalia, espina bífida, transposición de los grandes vasos y defectos ventriculares septales, finalmente, debe recalcarse que la mortalidad perinatal en diabéticas ha sido drásticamente reducida con el manejo moderno, pero que las malformaciones congénitas constituyen el principal determinante de la morbimortalidad perinatal, diabética, actual . Des de el punto de vista preventivo es fundamental señalar el hecho de que si las mujeres diabéticas se someten a programas de control pre-concepcional, con énfasis en la vigilancia de su glicemia, la tasa de malformaciones congénitas es similar a la de la población no diabética

Debemos siempre tener en mente que el período de mayor riesgo para anomalías congénitas de origen diabético es antes de la semana séptima postconcepción.

Los factores etiológicos en anomalías congénitas son todavía muy controversiales (Tabla 13), aunque se sigue considerando a la hiperglicemia como el factor principal por los posibles mecanismos señalados en la misma tabla.

TABLA. 13

FACTORES ETIOLOGICOS EN ANOMALIAS CONGENITAS DE INFANTES DE MADRES DIABETICAS

\author{
Hiperglicemia \\ Mecanismos: \\ -Daño al saco vitelino \\ -Mioinositol Intracelular reducido \\ -Deficiencia de ácido araquidónico \\ Hipoglicemia? \\ Inhibición de la somatomedina? \\ Vasculopatía materna? \\ Susceptibilidad Genética?
}

1-B. DETECCION PERINATAL DE MACROSOMIA FETAL Es la complicación fetal más frecuente de la diabetes. El diagnóstico clínico solo es practicable en pacientes delgadas y con una altura uterina previamente normal. De otro modo, debe efectuarse el ultrasonido cada 4 semanas a partir de la 20. Cuando una o más variables del tamaño fetal están por arriba del percentilo 95, particularmente la circunferencia abdominal o el peso fetal, el feto es probablemente macrosómico (18),

El manejo es controversial. Si el peso estimado es superior a $4,500 \mathrm{gm}$. amerita cesárea. Cuando el peso estimado es de 4,000 gm. a 4,500 gm. algunos autores recomiendan cesárea para evitar la probable distocia de hombros (19). En estos fetos macrosómicos, el conjunto de hombros y tronco puede ser mayor que la cabeza. Otros creen que en este caso no se justifica la cesárea, dado el respectivo error sonográfico en la estimación del peso fetal (20) y la baja incidencia de trauma fetal en esas condiciones, que es de 1/500. Aparentemente, lo aconsejable es permitir una labor espontánea y una conducta expectante donde al menor signo de distocia se recurra a la operación cesárea. En caso de posiciones cefálicas posteriores, persistentes, debe efectuarse también la cesárea.

1-C. PREVENCION DE HIPOXIA FETAL. Como se expresó anteriormente, a partir de la semana 34 debe efectuarse la vigilancia fetal preferentemente por el CST una vez por semana, y alternativamente, por el NST, dos veces por semana (21), o por el perfil biofísico una vez por semana. En los casos más severos, como las pacientes insulino dependientes inestables, o casos de retardo del crecimiento intrauterino se debe comenzar la vigilancia en la semana 28 .

\section{PERIODO DEL PARTO}

2-A CONTROL METABOLICO . En general, al momento de la labor y parto, los requerimientos de insulina disminuyen en la paciente con este tipo de terapia. Deben recibir su comida y dosis usual de insulina la noche anterior al parto o cesárea. El día del parto o cesárea no deben recibir la dosis matutina de insulina. En su lugar se les administra 50 $\mathrm{U}$ de insulina en $500 \mathrm{cc}$ de suero fisiólogo que se inicia a las 6:00 a.m. y se transfunde en infusión continua a la velocidad de $0.5 \mathrm{U}$ de insulina por hora. Esta dosis es ajustada de acuerdo a las glicemias medidas a cada hora. Esta infusión se descontinúa al terminar el parto.

2-B. MOMENTO DEL PARTO. En pacientes estables insulino-dependientes no es necesario adelantar la fecha del 
parto para antes de la semana 38. Pero al llegar la fecha estimada de parto sí se debe interrumpir el embarazo. Esto con el objeto de no agregar al embarazo diabético el riesgo del parto prolongado (22).

En pacientes insulino-dependientes inestables, el parto se debe efectuar tan pronto alcancen madurez pulmonar. Esto por cuanto las complicaciones fetales y maternas son grandes, por lo que es más riesgoso prolongar el embarazo más allá de dicha madurez pulmonar (23).

2-C. VIA DEL PARTO. La paciente insulino-dependiente estable, que llega al término de su embarazo puede tener parto vaginal si no hay contraindicaciones obstétricas.

La paciente insulino dependiente-inestable, cuyo embarazo se interrumpe al alcanzar madurez pulmonar fetal, puede tener muchas indicaciones de cesárea, pero hasta en un $50 \%$ pueden tener un parto vaginal seguro, si se vigilan estrictamente las glicemias maternas y la condición feto-placentaria.

\section{BIBLIOGRAFIA}

Siddigi T. Rossen B. Mimouri F. et al. "Hypertension During Pregnancy in Insulin Dependent Diabetic Women". Obstet Gynecol. $1991 ; 77:$ 514-519.

Kitzmiller Jl, Gin GB et al. "Preconception Care of Diabetes: GliCemic Control. Prevents Congenital. Anomalies". Jama 1991; 265 731-736.

arias F. "Practical Guide to High Risk Pregnancy and Delivery". 2ND ED, MosBY YB. 1993; 15: 280-298.

Coetz EJ, Jackson Wu, Berman PA. "Ketonuria In Pregnancy - with SPECIAL REFERENCE TO CALORIE RESTRICTED FOOD INTAKE IN OBESE DIABETES". DIABETES 1980; 29:177.

Maresh M, Gillmer MDG, Beard RW, et al. "The efFect of diet and INSULIN ON METABOLIC PROFILES OF WOMEN WITH GESTATIONAL DIABETES MELLITUS". DiABETES 1985; 34 (Supp.2): 88.

Jovanovich-Peterson L., Peterson CM. "Dietary manipulation as a PRIMARY TREATMENT STRATEGY FOR PREGNANCIES COMPLICATED BY DIABETES". J. AM COll NuTRIC 1990; 9: 320-325.

Jovanovich-Peterson L, Peterson CM: -Reed GF, et al. "Maternal. POST PRANDIAL GLUCOSE LEVELS AND INFANT BIRTH WEIGHT: THE DIABETES IN EARLY PREGNANCY STUDY". AM J OBSTET GYNECOL. 1991; 164:103-11

Kjos SL, Buchanan TA, Greenspoon JS, et al. "Gestational diabetes Melutus: THE PREVALENCE OF GLUCOSE INTOLERANCE AND DIABETES MELLITUS IN THE FIRST TWO MONTHS POSTPARTUM". AM J OBSTET. GYNECOL. 1990; 163:93-98.

Grant PT, OAts JN, Beischer N. "The long term follow-up of WOMEN With Gestational Diabetes". Aust NZ J Obstet GyneCol. 1986; Ó: 17 .
Skyler JS, Mintz DH O'Sullivan MJ. "Management of Diabetes And Pregnancy", in Rifkin H, Raskin P (eds): Diabetes Mellitus. Bowie MD, Robert J. Brady CO. 1981, Vol 5, Chap 19, pp 161-177.

Freinkel N. Dooley Sl, Metzger Be. "Care of the Pregnant Woman with Insulin-Dependent Diabetes Mellitus". N. Engl J Med 1985; 313:96-101.

Mintz DH Shyler JS, Chez RA. "Diabetes Mellitus and Pregnancy". DiABETES CARE 1978; 1:49-63.

Skyler JS, O'Sullivan MJ, Robertson DE, Skyler DL, Holsinger KK, Lasky IA, Mc Leod AG, Burkett G. Mintz DH. "Blood Glucose Control During Pregnancy". Diabetes Care 1980; 3: 69-76.

Leslie RDG, Pike DA, John PN, White JM. "Hemoglobin A1 in DiaBetic PREgNanCY". LanCet 1978; 2:958.

Mill JL, Baker L, Goldan as. "Malformations in Infants of Diabetic Mothers Occur Before the Seventh Gestational WeEk: Implications FOr Treatment". Diabetes 1979; 28: 292-293.

Reece EA, Hobbins JC. "Diabetic Embriopathy, Pathogenesis, Prenatal Diagnosis and Prevention". Obstet Gyecl Surv. 1986; 41: 325 335.

Gabbe SG. "Congenital Malformations in Infants of Diabetic motHers". OBSTEt GyneCOL SuRV. 1977; 32: 125-132.

Timor-Tritsch IE, Itskivotz J, Brandes JM. "Estimaton of Fetal Weight by Real Time Sonography". OBstat Gynecol 1981; 57 : 653 656.

Benedetti TJ, Gabbe SG; "Shoulder Dystocia: A Complication of Fetal Macrosomia and Prolonged Second Stage of labor with Midpelvic Delivfery". OBstet Gynecol 1978; 52: 526-529.

Tamura RK, Sabbagha RE, Dooley Sl et al. "Real Time Ultrasound Estimations of Weght in Fetuses of Diabetic Gravid mothers". AM J OBStET Gynecol 1985; 153-157.

Olofsson P. Sjoberg nO, Solum T. "Fetal Surveillance in Diabetic Pregnancy. II The Nonstress Test versus the Oxytocin Challengt Test". Acta Obstet. Gynecol ScCand 1986; 65 (4): 357.

Eden RL, Steifert LS, Winegar et al. "Maternal Risk Statesand Post date Pregnancy Outcome". J Reprod Med 1988; 33-53.

Rasmussen MJ, Firth R, Fooley M, Stronge JM. "The Timing of De livery in Diabetic Pregnancy: A 10 Year Review". Aust NZ Obstet GYNECOL 1992; 32 (4): 313.

Reece Ea, Coustan DR, Hayslett JP, et al. "Diabetic Nephropath Pregnancy Performance and Fetomaternal Outcome". Am J Obste GYNECOL 1988; 159: 56-66.

Klein BeK, Moss SE Klein R. "Effect of Pregnancy on Progressic of Diabetic Retinopathy". Diabetes Care 1990. 13: 34-40.

Silfin SL, Wapner RJ, Fabbe SG. "Maternal Outcome in Class H Di Betes Mellitus". ObStet. GyneCOL. 1980; 55: 749-751. 


\section{PERIODO POST PARTO}

CONTROL METABOLICO. En el período post-parto,ocurre una súbita disminución de la resistencia a la insulina. La mayoría de las pacientes no requieren insulina por 24-48 horas. Se deben efectuar glicemias en ayunas y post-prandiales. Tan pronto haya alteraciones de éstas, se debe restablecer la terapia insulínica utilizando de $1 / 2$ a $2 / 3$ de la dosis pre-parto, como promedio, y ajustándola a la respuestas del paciente.

\section{DIABETES INSULINO DEPENDIENTE CON DAÑO ORGANICO.}

V A. NEFROPATIA. Al principio, estas pacientes presentan leve proteinuria e hipertensión. En la segunda mitad del embarazo, ambas aumentan y aparece incremento de la creatinina sérica (24). A veces es difícil distinguirla de la pre-eclampsia. Las principales complicaciones son: prematuridad (45\%), y retardo del crecimiento intrauterino; éste debe ser diagnosticado temprano para evitar la muerte fetal. La longevidad de las pacientes diabéticas nefrópatas se reduce mucho, a menos que se haga transplante renal. También la nefropatía suele asociarse a retinopatía (80\%) pero solo el 20/ de ésta es de tipo proliferativo.

IV B. RETINOPATIA. Afecta al 40\% de todas las diabéticas insulino-dependientes y desgraciadamente, se acelera con el embarazo (25). El 20\% de éstas, tiene neovascularizaciones en la superficie de la retina, o sea, una retinopatía proliferativa. Estos nuevos vasos son frágiles y pueden sangrar con los cambios en presión intraocular que ocurren durante la labor de parto conduciendo a ceguera súbita. Por eso, la labor está contraindicada en estas pacientes.

IV C. CARDIOMIOPATIA. Produce frecuentemente mortalidad materno -fetal (26). Requiera el manejo en cuidado intensivo para mejorar el pronóstico.

\section{CONCLUSIONES}

Fisiopatológicamente la razón de que aparezca una intolerancia a la glucosa durante el embarazo, o de que en el curso del mismo se acentúe una diabetes pre-gestacional es que se aumenta la resistencia a la insulina. Esto es debido, por un lado a la destrucción de la insulina por los riñones y enzimas placentarias y, por otro, a la acción antagónica a la insulina del lactógeno placentario, la progesterona, el estradiol, y el cortisol de origen placentario.

Las embarazadas diabéticas tienen mayor incidencia de infección, pre-eclampsia, sangrado post-parto parto por cesárea.

La diabetes gestacional solo puede ser diagnosticada por valoración de todas las embarazadas con la prueba de sobre carga a la glucosa o la prueba de glicemia post-prandial. La incidencia de anomalías congénitas se puede abatir por control glicémico adecuado en el período periconcepcional. La glicemia debe ser mantenida por debajo de $105 \mathrm{mg} / \mathrm{dl}$. en ayunas y por debajo de $120 \mathrm{mg} / \mathrm{dl} 2$ horas post-prandial. Este último control es necesario en la prevención de la macrosomia fetal.

La diabética gestacional de alto riesgo debe tener vigilancia fetal a partir e la semana 34 y tener su parto entre las semanas 38-40.

La mayoría de los autores está de acuerdo en practicar cesárea cuando el peso total estimado excede $4.500 \mathrm{gm}$. El manejo es controversial si el peso varía de 4.000 gm. a $4.500 \mathrm{gm}$.

En el período post-parto la mayoría de las pacientes no necesita insulina por 24-48 horas, debido a la disminución de la resistencia a la insulina. 